\title{
Autonomic neuropathy in the development and progression of vascular complications of diabetes mellitus
}

\author{
Irina A Kurnikova*, Nikolay D Kisliy, Svetlana N Kislaya and Ramchandra Sargar \\ RUDN University, Moscow, Russia
}

\begin{abstract}
Background: Diabetic autonomic neuropathy is the most prevalent complication of diabetes. On the one hand, we perceive autonomic neuropathy as a complication of diabetes, on the other - as a factor of influence on the formation and progression of other complications of diabetes and, first of all, from the cardiovascular system.

Methods: the study of the state of regulatory mechanisms in the conditions of formed cardiac autonomic neuropathy was carried out. The absolute and relative values of heart rate variability indices were studied the calculated coefficients (vegetative sympathetic balance, centralization index) in patients with diabetes mellitus.

Results: impairment of mechanisms of vegetative regulation in the conditions of formed diabetic cardiac autonomic neuropathy, manifested by changes in the structure of the frequency characteristics of the wave spectrum in each age group compared with healthy people, were revealed. Spectral analysis showed that the level of regulation corresponded to the parameters of patients of the older (for a decade) age group. The obtained results allow to explain the appearance of structural changes in the vascular wall due to atherosclerosis (this condition is called macroangiopathyin diabetic patients) in young patients.

Conclusions: Diabetic autonomic neuropathy is a common complication of diabetes, which leads toimpairment of the mechanisms of regulation at the level of the whole organism, and to the progression of vascular complications. It is proved that an important component contributing to the early development and rapid progression of cardiovascular diseases in diabetic patients, in addition to endothelial dysfunction, is vegetative disregulation. The "aging of regulatory systems" in patients with diabetes mellitus with cardiac autonomic neuropathy occurs 10 years earlier than the biological age of the patient and, consequently, earlier "switch on" the mechanisms of atherogenesis.
\end{abstract}

Abbreviations: ANS - autonomic nervous system; CAN - cardiac autonomic neuropathy; DAN - diabetic autonomic neuropathy.

\section{Background}

The autonomic nervous system is one of the most important regulatory mechanisms for adequate functioning of the human body, one of the main components of the body adaptation to external and internal environment. Insulin deficiency or its excess in diabetes leads to the primary lesion of the nervous tissue and to the activation of additional resources of the body to preserve internal homeostasis, which requires the switching of the regulation system to a higher and energy-consuming level (Figure 1).

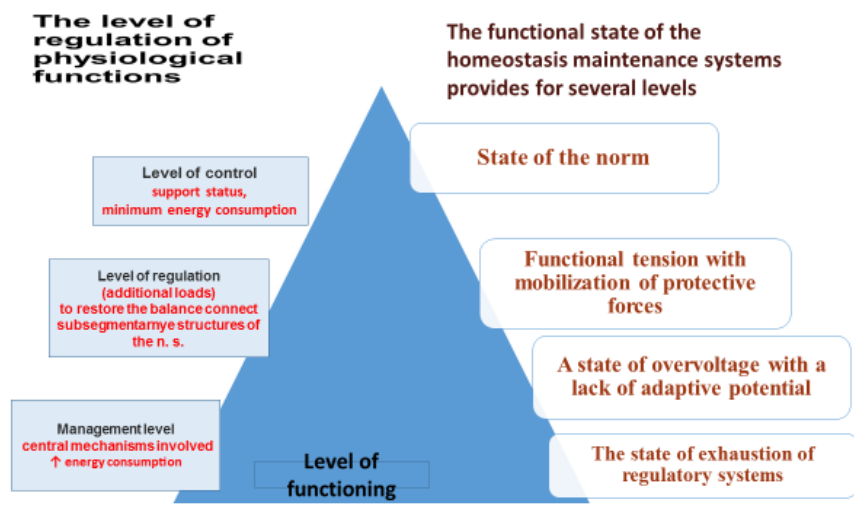

Figure 1. Regulatory systems of the body
Autonomic nervous disorders occur in a large number of people, even who considerthemselves healthy. In clinical practice, the term dysautonomia or autonomic dysfunction (AD) is often used to characterize such disorders. The clinical symptoms of autonomic dysfunction are very different - lightheadedness, tremulousness and presyncope, heart-rhythm variability with irregular beats or fast heart rate, shortness of breath, poor exercise tolerance, lack of saliva, dysfunction gastrointestinal system, lack of sweating or excessive sweating, temperature regulation disturbance, stressed, anxious etc. The reason is the imbalance between sympathetic and parasympathetic systems. In population-based epidemiological studies, vegetative disorders occur in $30-80 \%[1,2]$, the risk increases in the presence of diabetes mellitus [3-5]. In patients with DM with cardiac autonomic neuropathy (CAN) mortality within the next 5 years is $50 \%$ [5-7].

Change in the function of the nervous system, as a consequence of metabolic disorders, develops in parallel with the progression of diabetes. Signs of diabetic autonomic neuropathy (DAN) are found in $3.5-6 \%$ of patients at the onset of the disease, and in $100 \%$ of patients with prolonged duration of diabetes mellitus [5,6]. Insulin deficiency

${ }^{*}$ Correspondence to: Irina A Kurnikova, RUDN University, Moscow, 117198, Russian Federation, Tel: +7 (926) 479-24; E-mail: curnikova@yandex.ru

Key words: diabetes mellitus, autonomic nervous system, autonomic dysfunction, cardiac autonomic neuropathy, diabetic autonomic neuropathy, functional body reserves, autonomic regulation

Received: September 15, 2018; Accepted: September 25, 2018; Published: September 28, 2018 
leads to dysregulation of all physiological systems. Insufficient supply of energy substances, first of all, glucose into the cell associateswith hypoxia [8,9]. The process can occur at different speeds and lead to the dystrophy and apoptosis. Sensitivity to the lack of nutrients and oxygen is determined by the type of tissue and primarily affects those that carry out regulatory functions (brain, lungs, myocardium, intestines). In case of oxidative stress, the phospholipid structure of membranes is damaged, proapoptotic proteins are formed, metabolic processes decreases, dystrophic changes and autoimmune reactions develop [911]. The functions of the autonomic nervous system are impaired.

What significance does the vegetative system have in regulating the activity of an organism? The most important system in the regulation of the relationship between the organism and the environment. It provides our existence in a rapidly changing environment. Assessment of vegetative status includes:

- Vegetative tonus - is the tonic equivalence of the sympathetic and parasympathetic divisions of the N.S. (eutony) or the predominance of one of them;

- Vegetative reactivity - is a dynamic (amplitude-time) characteristic of the processes of regulation of the integrative system of the autonomic functions of the organism (response to stimuli);

- Vegetative provision - quantitative and qualitative characteristics of vegetative conditions to ensure the necessary level of life in general and for specific organs and systems.

Diabetic autonomic neuropathy (DAN) is manifested by vegetative disregulation, which, in turn, aggravate $\mathrm{DM}$, promotes the carbohydrate absorption variability, disruption of the counterregulation in hypoglycemia, progression of vascular complications, the formation of osteoarthropathy and ulcerative defects, reduces the bioavailability of oral hypoglycemic drugs, affects the heart rate and blood pressure $[6,12]$.

The most important factor in assessing the state of ANS as a whole is the functional state of the cardiovascular system. The modern methods allows to diagnose CAN at early stage, the main clinical manifestations of CAN are: persistent tachycardia; weak reaction to drugs; declining of R-R intervals; increased sensitivity to catecholamines (arrhythmia, asystole); silent myocardial infarction; in cases of diminishingsympathetic innervationslike fixed heart rate; orthostatic hypotension; "cardiopulmonary arrest."

Various methods are used to study the state of vegetative regulation: "questionnaires to identify signs of vegetative changes", "study schemes to identify signs of vegetative disorders", variation pulsometry, $24 \mathrm{hr}$ monitoring of blood pressure and heart rate $[1,13]$. The change of heart rate variability (HRV) is one of the most sensitive tests for early and preclinical diagnosis of CAN.

As already mentioned, the onset of CAN changes the course of diabetes, accelerates the development and progression of vascular complications. Despite the obvious connection between these phenomena, the mechanisms of the influence of autonomic dysfunction on the rate of progression of vascular complications are not fully known. Traditionally, the mechanisms of vascular complications are considered only from the standpoint of metabolic disorders. Our study allowed us to establish an additional and, in our opinion, a very important pathogenetic mechanism - the effect of autonomic dysfunction on the development of vascular complications in patients with diabetes mellitus.
Purpose of study: to study clinico-functional assessment of the state of vegetative regulatory mechanisms and the risk of developing vascular complications in patients with diabetes

\section{Methods}

\section{Design}

The study was conducted on the medical center of RUDN University (Moscow) - based on the Endocrinology Department of City Clinical Hospital. A.K. Eramishantseva (Moscow) from 2015 to 2018. The survey included 513 patientswith a diagnosis of diabetes mellitus, aged 20 to 60 years. It was a single-stage epidemiological study (crosssectional observational study) with an emphasis on the state of indices of vegetative regulation. Individual groups of patients underwent dynamic observation.

\section{Sampling and Setting}

The study comprised 513 individualsand had 2 stages. At the first stage, the incidence of cardiac autonomic neuropathy was analyzed in 513 patients admitted to the endocrinology Department. At the second stage, 101 patientswith diabetes mellitus and 37 healthy volunteers underwent a comparative analysis of heart rate variability as well as the relationship with the main complications of diabetes. Patients of each group were divided into subgroups according to age: $20-30$ years, $31-40$ years, $41-50$ years, $51-60$ years (Table 1 ).

Exclusion criteria - Age younger than 20 years, patients admitted in a state of ketosis or ketoacidosis, acute cardiovascular disorders. The basis for including patients in the study was also the informed consent of the patient.

\section{Ethical consideration}

The study was conducted in compliance with the principles of medical ethics. The compliance of the study with the norms of biomedical ethics was confirmed by the conclusion of the Ethics Committee of the Medical Institute of the Peoples' Friendship University of Russia (Protocol №. 9 of Marth 17, 2016). Participants were fully acquainted with the objectives of the study, its importance, and the method of selecting participants, the right to refuse to participate at any time, the benefits and risks of the study.

\section{Instruments and data collection procedure}

Glucose level was controlled according to WHO recommendations: Glucose profile; Glycated Hemoglobin (HbAlc) [14]. Criteria of compensatory DM type 2 are determined according to European Association for the Study of diabetes [2015].

The main method was assessment of 24 hours heart rate variability on the apparatus "Valenta", equipped with a program for computer processing of spectral analysis and time analysis.

ECG was registered in allpatients with in 1-5 modified thoracic leads within 24 hours. During the study, the patient kept a "diary". Subsequent analysis was performed automatically in relation to the patient's physical and other activities. Heart rate indicators (day, night and average daily) were monitored. The circadian dynamics of ECG parameters was studied: the time of atrioventricular conduction was determined by the value of the PR interval and the circadian dynamics of the QRS complex. Time domain (HRV) analysis included: SDNN (ms) - the square root of the standard deviation of all N-N intervals; RMSSD (ms) - the square root of the mean square of the differences of successive N-N intervals; pNN50 (\%)-the ratio of the intervals N-N, 
Table 1. Age spectrum of frequency ranges in patients with diabetes mellitus

\begin{tabular}{|c|c|c|c|c|}
\hline \multirow{2}{*}{ Index } & Age 20-30 л. & Age 31-40 л & Age 41-50 л & Age 51-60 л \\
\hline & $(n=19)$ & $(n=24)$ & $(\mathrm{n}=\mathbf{2 1})$ & $(n=37)$ \\
\hline $\mathrm{CI}$ & $130,2 \pm 11,1 * *$ & $132 \pm 9,6^{*}$ & $125,5 \pm 3,7^{*}$ & $119,4 \pm 9,8^{*}$ \\
\hline H.Rd.med. & $87,4 \pm 1,9^{*}$ & $87,8 \pm 7,4^{*}$ & $81,9 \pm 6,1^{*}$ & $84,0 \pm 12,1^{*}$ \\
\hline SDNN $\left(\mathrm{Mc}^{2}\right)$ day & $31,1 \pm 7,7 * *$ & $0,021 \pm 0,01 * *$ & $0,031 \pm 0,01 * *$ & $0,042 \pm 0,01^{*}$ \\
\hline SDANN $\left(\mathrm{Mc}^{2}\right)$ day & $57,9 \pm 14,5^{*}$ & $0,05 \pm 0,01 * *$ & $0,052 \pm 0,01 * *$ & $0,081 \pm 0,02^{* *}$ \\
\hline pNN $50 \%$ & $6,6 \pm 0,9^{* *}$ & $8,4 \pm 0,3^{*}$ & $4,7 \pm 0,2$ & $5,4 \pm 0,2$ \\
\hline $\mathrm{TF}\left(\mathrm{mc}^{2}\right)$ & $6155,6 \pm 54,6$ & $3978,3 \pm 41,9^{*}$ & $5621,3 \pm 67,4^{*}$ & $4437,8 \pm 27,4^{*}$ \\
\hline ULF (\%) & $20,7 \pm 3,1^{*}$ & $56,6 \pm 7,4$ & $47,9 \pm 3,1^{*}$ & $41,0 \pm 4,2^{*}$ \\
\hline $\mathrm{ULF}\left(\mathrm{mc}^{2}\right)$ & $2236,9 \pm 29,8^{* *}$ & $2383,2 \pm 31,7$ & $2502,5 \pm 28,5^{* *}$ & $1945,9 \pm 29,5^{*}$ \\
\hline VLF (\%) & $31,9 \pm 7,2$ & $27,3 \pm 2,4$ & $23,4 \pm 7,7$ & $23,0 \pm 3,7$ \\
\hline $\operatorname{VLF}\left(\mathrm{mc}^{2}\right)$ & $3607,5 \pm 44,1^{* *}$ & $864,7 \pm 36,3$ & $1393,9 \pm 21,4^{*}$ & $1636,9 \pm 21,8 *$ \\
\hline LF (\%) & $28,4 \pm 3,7$ & $6,75 \pm 1,0^{* *}$ & $13,3 \pm 3,8^{*}$ & $18,9 \pm 7,2^{*}$ \\
\hline $\mathrm{LF}\left(\mathrm{mc}^{2}\right)$ & $3751,8 \pm 91,0^{*}$ & $289,2 \pm 22,7$ & $880,3 \pm 12,1$ & $1400,1 \pm 61,4 *$ \\
\hline HF (\%) & $18,96 \pm 2,1$ & $9,36 \pm 0,9^{*}$ & $15,2 \pm 2,1^{*}$ & $17,3 \pm 4,4^{*}$ \\
\hline $\mathrm{HF}\left(\mathrm{mc}^{2}\right)$ & $2559,6 \pm 29,1^{* *}$ & $460,7 \pm 27,0$ & $987,4 \pm 31,9^{*}$ & $1161,7 \pm 17,9^{*}$ \\
\hline $\mathrm{LF} / \mathrm{HF}$ & $1,77 \pm 0,7$ & $1,11 \pm 0,4$ & $0,96 \pm 0,03 *$ & $1,09 \pm 0,03^{*}$ \\
\hline Index & $\begin{array}{c}\text { Age20-30 л. } \\
(\mathrm{n}=19)\end{array}$ & $\begin{array}{l}\text { Age } 31-40 \text { л } \\
(\mathrm{n}=24)\end{array}$ & $\begin{array}{l}\text { Age41-50 л } \\
(\mathrm{n}=21)\end{array}$ & $\begin{array}{c}\text { Age } 51-60 \text { л } \\
(\mathrm{n}=37)\end{array}$ \\
\hline
\end{tabular}

Indicators that have deviations from the age norm are marked: * ${ }_{-}<0,05 ; * *-p<0,01$

different from the neighboring more than $50 \mathrm{~ms}$, to the total number of intervals N-N.

For the quantitative characteristic of periodic heart rate processes, the spectral analysis of HRV (frequence domain) was carried out with the division of the processed sample of RR intervals into frequency spectra of different densities by means of fast Fourier transform. Spectral parameters were analyzed:

* SP $\left(\mathrm{ms}^{2}\right)$ - total spectral power of neurohumoral regulation $\left(\mathrm{ms}^{2}\right)$ in the range of $0.003-0.40 \mathrm{~Hz}$;

* LF $\left(\mathrm{ms}^{2}\right)$ - low frequency component of the spectrum - waves 0.04$0.15 \mathrm{~Hz}$, reflects the activity of the sympathetic system;

${ }^{*} \mathrm{HF}\left(\mathrm{ms}^{2}\right)$ - high frequency component of the spectrum - waves from 0.15 to $0.40 \mathrm{~Hz}$, the activity of the parasympathetic nervous system at the segmental level;

* $\operatorname{ULF}\left(\mathrm{ms}^{2}\right)$ - ultra low frequency component of the spectrum - waves up to $0.0033 \mathrm{~Hz}$, the powerof the waves ultra-low frequency reflects the activity of the higher centers of heart rhythm;

${ }^{*} \operatorname{VLF}\left(\mathrm{ms}^{2}\right)$-Very low frequency component of the spectrum humoralmetabolic-0.0033-0.04 Hz, reflects the functional state of the suprasegmental structures.

\section{And two calculated index:}

Index of Centre (IC) - Ratio of the activity of the central regulation loop to the autonomous $=(\mathrm{LF}+\mathrm{VLF}) / \mathrm{HF}$; LF/HF

Index vagosympathetic balance (IVB) - ratio between LF \& HF =

In our studies the analysis was carried out automatically. Methods of mathematical transformations with the subsequent physiological and clinical assessment of value of the received parameters were usedfor the analysis of variability.

Daily monitoring of arterial blood pressure (DMABP) was carried out on the device "BP LAB" (N. Novgorod) with the definition of 4 groups of main indicators: mean, median, mode of BP; pressure load index - time index and area index; indicators of the daily rhythm of BP: the degree of night decrease in BP (DNDBP) for systolic and diastolic
$\mathrm{BP}$; indicators of variability of $\mathrm{BP}$ - standard deviation from the mean $\mathrm{BP}$ (SD). Additionally: the maximum values of $\mathrm{BP}, \mathrm{BP}$ increase in the morning compared to the night hours, the rate of increase in $\mathrm{BP}$, the presence of "peak" values of BP, the integral index of trouble (IIT), hypotension time index.

\section{Data analysis procedures}

Processing of received data - Program STATISTIC 10,0 (Matematica, Matlab ${ }^{\circ}$ Harvard Graphics ${ }^{\circ}$ ) Stat Soft). Descriptive statistical measures including frequency and percentages were used to describe.

\section{Results}

According to our obtained data at the first stage of examination of 513 patients, the prevalence of autonomic neuropathy, which was diagnosed by the results of $24 \mathrm{~h}$ monitoring of the heart rate with subsequent spectral and temporal analysis, were very high, especially in the cardiovascular form (Figure 2). Regardless of the type of DM, the quality of glycemic control significantly influenced on the formation and activity of the cardiovascular form of autonomic neuropathy: in patients with type $1 \mathrm{DM}$ and optimal indices of metabolic compensation of carbohydrate metabolism (HbAlc) CAN was registered 3.5 times less than in patients who did not reach the target values of Hbalc, in patients with type 2 diabetes-1.3 times less (Figure 2).

Diabetic decompensation was accompanied by a significant change in heart rate variability (HRV), the direction and severity of impairments in patients with different types of diabetes was different. Thus, the circadian index $(\mathrm{CI})$ in patients with type $2 \mathrm{DM}$ was very low $1.19 \pm 0.08$, which is possible only with a pronounced damage of central and vegetative link of the regulation of the heart rate. The power of low-frequency spectrum (LF-24,2 $\pm 2,1 \%$, VLF-23,4 $\pm 3,7 \%$, ULF-30,8 $\pm 8,4 \%$ ) prevailed over the power of high-frequency (HF-21,6 $\pm 2,9 \%$ ), which is typical for pronounced autonomic dysfunction and disruption of autonomic regulation. The age of the patient and the duration of diabetes were not of fundamental importance. Decompensation of type $1 \mathrm{DM}$ did not affect CI $(1.32 \pm 0.17)$ but was accompanied by a decrease in the total spectral power (TS $1675 \pm 233$ MS2), the predominance of low-frequency spectrum power (ULF- $36.5 \pm 11 \%$, VLF-33.9 $\pm 4.7 \%$, LF-17.4 $\pm 6.9 \%$ ) over high-frequency (HF-11.7 $\pm 1.5 \%$ ). The coefficient of vagosympathetic balance in patients with type $1 \mathrm{DM}$ on the 


\section{PREVALENCE OF CARDIOVASCULAR FORM OF DIABETIC AUTONOMIC NEUROPATHY IN DIAGNOSED PATIENTS}

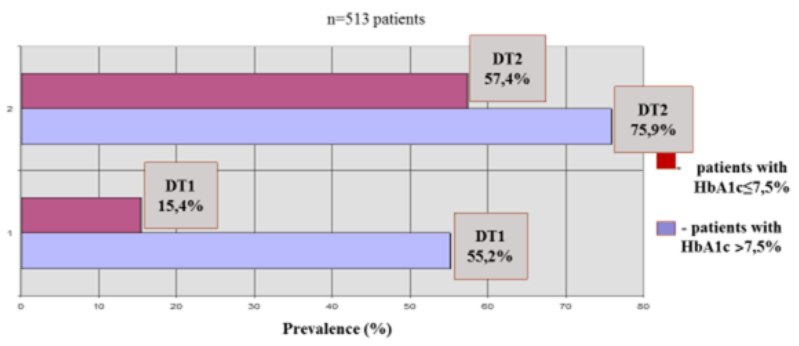

Figure 2. Prevalence of cardiovascular form of diabetic autonomic neuropathy. DT1 diabetes type 1; DT2 - diabetes type 2; HbA1c - glycated hemoglobin

\section{Indices of the state of autonomic regulation}

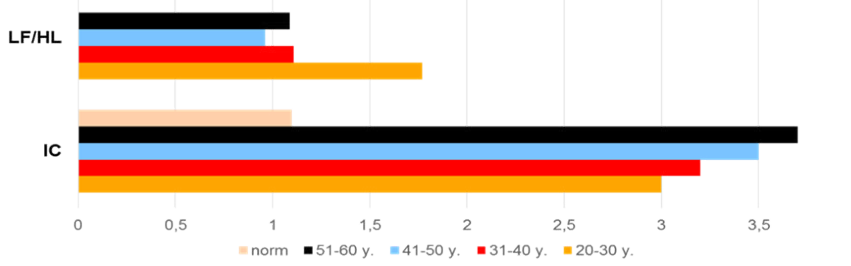

Figure 3. Indices of the state of autonomic regulation. LF/HF - Index vagosympathetic balance (ratio between LF \& HF); IC - Index of Centre (ratio of the activity of the central regulation loop to the autonomous)

background of decompensation (LF/HF) was higher than the average - $1.58 \pm 0.4$, confirming hypersympathicotonia. In patients with type 2 diabetes mellitus decompensation was also accompanied by vegetative imbalance but proceeded against the background of increased activity of the parasympathetic nervous system $(\mathrm{LF} / \mathrm{HF}=0.86 \pm 0.07)$.

In the second stage, we compared the frequency characteristics of daily monitoring of heart rate in patients with DM and healthy people in similar age groups (Table 1).

The obtained data indicates significant abnormality of the regulation of the heart rate due to diabetic cardiac neuropathy in almost all patients, regardless of age. Heart rate in each age group was about $10-12 \%$ higher than the average age. There was a decrease in all indicators of the time analysis. The increase in the VLF spectrum, indicating the activation of Central regulation, confirmed the tension of adaptive mechanisms transferred by the body from the level of control to the level of centralization. Also, the extreme tension of adaptation and even disruption of autonomic regulation is indicated by the data on the increase of ULF in patients, starting age from 30 years. Compared with the average age, the changes in the groups are very significant. In young patients, activation of both sympathetic and parasympathetic systems was observed, but hypersympathicotonia prevailed, so the LF/ HF ratio was also increased.

At the age of 30-40 years, instead of the predominance of parasympathetic activity and a decrease in sympathetic activity, we saw in our patients a decrease in parasympathetic activity, which is more typical for patients of the next decade of life. Abnormal vegetative balance was accompanied by simultaneous activation of two systems of multidirectional action, which led to a rapid depletion of the system of regulation of the heart rate and adaptive capacity of the body.

Since the age of 41 , an active centralization of regulative mechanisms has been observed (increase in VLF), which usually commences 1015 years later. Signs of autonomic dysfunction were observed in most patients, a decrease in TF on the background of decompensation indicated a decrease in the reserve capacity of the patient's body.

The Circadian Index (CI) in the majority of patients with type 2 diabetes was very low $-1.19 \pm 0.08$, which is possible only with a marked impairment of the central and vegetative counter regulating of heart rate. The coefficient of the vagosympatetic balance of patients with type 1 diabetes of non-compensation ( $\mathrm{LF} / \mathrm{HF})$ was above the average of $1.58 \pm 0.4$ (midl $1.77 \pm 0.7$ ), confirming hypersympathetic tonus. In patients with type 2 diabetes, non-compensation also accompanied by vegetative imbalance, occurred against a background of increased activity of the parasympathetic nervous system $(\mathrm{LF} / \mathrm{HF}-0.96 \pm 0.03)$ (Figure 3).

In the study of BP monitoring findings, the average time indicators that do not have a reliable connection with the type, diseases duration and compensation of diabetes, was immediately turned outinattentively. The most important indicators were the variability of BP (VSBP day, VSBP night, VDBP day, VDBP night) and area indices at night (AI SBP night, AI DBP night) (systolic blood pressure - SBP; Diastolic blood pressure - DBP; index time - IT; morning pressure rise rate - MPRR; area index - AI). The reduction of VSBP, VDBP and index square in the night time in diabetic patients demonstrated the progression of the disease. Indicators of pressure load (IP) at night have additional diagnostic value in the diagnosis of diabetes, as it characterizes the safety of functional reserves of the patient.

\section{Discussion}

Structural disorders in the nervous tissue in patients with diabetes are quite common and the main mechanisms of their development are well known $[5,15,16]$. However, the question of the influence of autonomic regulation disorders on the rate of progression of vascular complications remains unanswered $[4,16,17]$. As a rule, the discussion takes place at the level of the primacy of the influence of vasa nervorum and nervivasorum. However, not only molecular mechanisms can be important, but also disorders that occur at the level of the whole organism, since the autonomic nervous system is responsible for adaptation and regulates the functions of all other systems.

The unity of the regulation system is always preserved-the mobilization of energy and metabolic resources is activated through nervous and humoral mechanisms [1].

There are 3 levels of activity of regulatory systems: control level; regulation level and management level. Work at the level of control requires minimal costs; it is to monitor the state of the regulated system without interfering in its work. With the appearance of additional loads, the regulation mechanism includes a higher level, which allows the body to provide an additional amount of energy and restore balance. If these resources are insufficient, the regulation system switches to the management level, connect to higher levels of Central control.

Subcortical neural centerequilibrates parameters within systems, providing intra-system autonomic balance [1]. Higher autonomic centers equilibrate system of the body among themselves, providing hormonal and vegetative homeostasis. The Central nervous system, 
including the cerebral cortex, coordinates the activities of all systems of the body in accordance with changes in the environment. Important in the assessment of adaptive capacity of vegetative homeostasis is the balance between the sympathetic and parasympathetic parts of the autonomic nervous system.

As a principle, the activity of the parasympathetic system is prevailed in young people up to 30 years, at the age of 31 to 40 years, these systems are equilibration, and in the fifth decade of life the activity of the sympathetic system is gradually increased. When the characteristics of spectral analysis has been compared, observed that the structure of the spectrum in diabetic patient (the correlation of HF, LF, ULF, VLF) does not appropriate to the indicators of the age of control group, but it appropriates to the indicators of the control group of the next (10 years older) group. This has been confirmed by the changes in the vagosympathetic balance (LF/HF). Simultaneously, the enhancement of subcortical centers and the transition of regulation from the level of control to the level of management has been observed. The inclusion of more energy-intensive regulatory mechanisms naturally led to a rapid depletion of functional reserves. All this we have considered as the manifestations of the" early aging" of the autonomic regulatory system. And allowed to explain why patients with diabetes with cardiovascular disease are observed not only more often than in the General population, but also appear earlier (at a younger age).

As a result of the conducted research has been defined that the tone and reactivity of the autonomic nervous system changes in diabetes mellitus. The depletion of energy resources and the failure of adaptation lead to early aging of regulatory mechanisms that manifests itself at the level of the whole organism by non-physiological early start of atherosclerosis. And we believe that this mechanism can be considered as a component in the pathogenesis of vascular lesions in DM patients along with endothelial dysfunction.

\section{Conclusion}

In General, we can say that the "aging of regulatory systems" in patients with diabetes mellitus with cardiac autonomic neuropathy occurs 10 years earlier than the biological age of the patient. The obtained data confirms the hypothesis that one of the most important reasons for the early onset of atherosclerosis and associated pathology of the cardiovascular system is in addition to endothelial dysfunction and the phenomenon of "early aging of regulatory systems".

The study of heart rate variability indices allows not only to identify already existing disorders with the system of regulation and adaptation, but also to predict the risk of developing diseases by measuring the overall stress of regulatory mechanisms, neurohumoral regulation of the heart and the relationship between sympathetic and parasympathetic parts of the autonomic nervous system.

Similar disorders can be observed in patients with diabetes mellitus, complicated by autonomic neuropathy. However, the interest is also the question - how early these disorders start to manifest, what changes in the state of vegetative regulation are observed in patients with metabolic syndrome and how much it leads to an increased risk of developing cardiovascular diseases.

There is no doubt that early (or preventive) treatment of diabetic autonomic neuropathy will also be a means of preventing cardiovascular diseases in patients with diabetes mellitus.

\section{Authors' contributions}

Irina Kurnikova was a major contributor in writing the manuscript and analyzed and interpreted the data of patients. Svetlana Kislaya participated in the examination of patients and collection of material. Nikolay Kisliy contributed to manuscript preparation. Ramchandra Sargar participated in collection of material and manuscript preparation. All authors read and approved the final manuscript.

\section{Acknowledgments}

This work was supported by RUDN University.

\section{References}

1. Autonomic Disorders: Clinical Picture, Diagnostics, Treatment / ed. A.M. Wayne. M. "MedicalNewsAgency", 2003. 752? pp: 109-118; 124-125.

2. Morales J (2014) Use of heart rate variability in monitoring stress and recovery in judo athletes. J. Morales [Ed]. Clin Physiol Funct Imaging 34: 230-236.

3. Dimitropoulos G, Tahrani AA, Stevens MJ (2014) Cardiac autonomic neuropathy in patients with diabetes mellitus. World J Diabetes 5: 17-39.

4. Aggarwal S, Tonpay PS, Trikha S, Bansal A (2011) Prevalence of Autonomic Neuropathy in Diabetes Mellitus. Current Neurobiology 2: 101-105.

5. Vinik AI, Erbas T (2013) Diabetic autonomic neuropathy. Handb Clin Neurol 117: 279294. [Crossref]

6. Ziegler D (1999) Cardiovascular autonomic neuropathy. Clinical manifestations and measurement. Diabetes Rev 7: 342-357.

7. Hoeldtke RD, Bryner KD, VanDyke K (2011) Oxidative stress and autonomic nerve function in early type 1 diabetes. Clin Auton Res 21: 19-28. [Crossref]

8. Baynes HW (2015) Classification, Pathophysiology, Diagnosis and Management of Diabetes Mellitus. J Diabetes Metab 6: 541 .

9. Ziegler D, Buchholz S, Sohr C, Nourooz-Zadeh J, Roden M (2014) Oxidative stress predicts progression of peripheral and cardiac autonomic nerve dysfunction over 6 years in diabetic patients. Acta Diabetol pp: 014-0601-3

10. de M Bandeira S, da Fonseca LJ, da S Guedes G, Rabelo LA, Goulart MO (2013) Vasconcelos SM. Oxidative stress as an underlying contributor in the development of chronic complications in diabetes mellitus. Int J MolSci 14: 3265-3284.

11. Hoeldtke RD, Bryner KD, VanDyke K (2011) Oxidative stress and autonomic nerve function in early type 1 diabetes. Clin Auton Res 21: 19-28. [Crossref]

12. Dimitropoulos G, Tahrani AA, Stevens MJ (2014) Cardiac autonomic neuropathy in patients with diabetes mellitus. World J Diabetes 5: 17-39. [Crossref]

13. Danieli A, Lusa L, PotoÄŤnik N, Meglï̈ॅ̆ B, Grad A, et al. (2014) Resting heart rate variability and heart rate recovery after submaximal exercise. Clin Auton Res 24: 5361. [Crossref]

14. World Health Organization: Definition, Diagnosis and Classification of Diabetes Mellitus and its Complications: Report of a WHO Consultation. Part 1: Diagnosis and Classification of Diabetes Mellitus (WHO/NCD/NCS/99.2). Geneva: World Health Org; 1999.

15. Albers JW, Pop-Busui R (2014) Diabetic neuropathy: mechanisms, emerging treatments, and subtypes. Curr Neurol Neurosci Rep 14: 473

16. Spallone V, Ziegler D, Freeman R (2011) Toronto Consensus Panel on Diabetic Neuropathy. Cardiovascular autonomic neuropathy in diabetes: clinical impact, assessment, diagnosis, and management. Diabetes Metab Res Rev 27: 639-653

17. Gaede P, Vedel P, Larsen N, Jensen GV, Parving HH, et al. (2003) Multifactorial intervention and cardiovascular disease in patients with type 2 diabetes. $N$ Engl $J$ Med 348: 383-393. [Crossref]

Copyright: (C2018 Kurnikova IA. This is an open-access article distributed under the terms of the Creative Commons Attribution License, which permits unrestricted use, distribution, and reproduction in any medium, provided the original author and source are credited. 